\title{
High-energy diode-pumped alexandrite amplifier development with applications in satellite-based lidar
}

\author{
Alexander T. Coney* and Michael J. Damzen \\ Photonics Group, Blackett Laboratory, Imperial College London, SW7 2AZ, UK \\ *alexander.coney11@alumni.imperial.ac.uk
}

\begin{abstract}
Efficient, wavelength-tunable diode-pumped alexandrite laser systems offer the potential for a more versatile, satellite-based lidar source compared to fixed wavelength Nd:YAG systems and non-space compliant lamp-pumped alexandrite. In this paper, we develop a strategy to enable the high-energy operation required for atmospheric lidar based on an efficient diode-pumped Master-Oscillator Power-Amplifier (MOPA) system design. A novel multi-pass 'diamond' slab amplifier geometry is introduced alongside the experimental results of the world's first diode-pumped alexandrite amplifier producing a gain of 2.13 in a demonstration system. A diode-pumped Q-switched alexandrite oscillator is presented with a record-highest pulse energy of $3.80 \mathrm{~mJ}$. Detailed optimisation of a two-stage amplifier design is studied numerically and maximised with temperature, wavelength and pump pulse duration to produce $50 \mathrm{~mJ}$ pulse energy. This forms part of an optimised alexandrite MOPA design capable of high pulse energy, showing the future potential of diode-pumped alexandrite for satellite-based atmospheric lidar.
\end{abstract}

(c) 2020 Optical Society of America

\section{Introduction}

Satellite-based atmospheric remote sensing enables accurate weather predictions, climate change observations and detailed atmospheric topography. Space-based systems require reliable and efficient high-energy (50 - $100 \mathrm{~mJ})$ laser pulses typically tens of nanoseconds in duration at around $100 \mathrm{~Hz}$ pulse rate [1]. Current systems are based on Nd:YAG and are limited to the wavelengths of $1064 \mathrm{~nm}$ and other harmonics (532 nm, $355 \mathrm{~nm}$ etc.) [1]. This prevents effective use of techniques such as resonance scattering lidar or DIAL (differential absorption lidar). Optical parametric oscillators (OPOs) offer wavelength tunability but add undesirable complexity for the space environment and low efficiency when requiring an Nd:YAG pump laser with harmonic conversion. Diode-pumped alexandrite lasers offer a compact, tunable alternative $(700 \mathrm{~nm}$ $858 \mathrm{~nm}$ ) [2,3], capable of targeting the absorption lines of oxygen (760 $\mathrm{nm}$ [4]), water vapour (730 nm [5], [6]) and free metal atoms such as potassium (770 nm [7]) and iron (386 nm [8], through second harmonic generation). Satellite-based alexandrite lasers would enable a more comprehensive map of the atmosphere, particularly in the mesosphere and lower thermosphere $(80-110 \mathrm{~km})$ where free atoms exist $[9,10]$.

Alexandrite $\left(\mathrm{Cr}^{+}: \mathrm{BeAl}_{2} \mathrm{O}_{4}\right)$ has a combination of high thermal conductivity $\left(23 \mathrm{Wm}^{-1} \mathrm{~K}^{-1}[2]\right)$ and long upperstate lifetime ( $262 \mu \mathrm{s}$ [2] at $\left.22^{\circ} \mathrm{C}\right)$ making it an ideal candidate for high-energy Q-switched operation. Advancements in $\mathrm{AlGaInP}$ (red) diode laser technology has renewed interest in diode-pumped alexandrite enabling the production of efficient, reliable and compact systems [11], [12], [13], [14]. Recent work with LED-pumped Ce:YAG concentrators [15] for pumping alexandrite has also been demonstrated, although greater efficiency is necessary for satellite-based operation.

Whilst significant energy storage is possible within alexandrite, the emission cross section is over an order of magnitude less than that of Nd:YAG. This requires intensive pumping which leads to high inversion and significant pump-induced lensing [16] in a high-energy system. A high pulse 
energy $\mathrm{TEM}_{00}$ oscillator is demanding, especially when combined with very narrow linewidth (< $30 \mathrm{MHz}$ for potassium resonance scattering lidar [17]). A Master-Oscillator Power-Amplifier (MOPA) strategy offers a better balance using a moderate (multi-mJ) oscillator with high spatial and spectral quality and a subsequent enhancement in energy within an amplifier system.

This paper describes the various aspects of amplifier design and optimisation, presenting work from the world's first diode-pumped alexandrite amplifier and a record-energy diode-pumped Q-switched alexandrite oscillator. The amplifier design developed is the multi-pass 'diamond' geometry which is based on a variation of the bounce [18] and folded zig-zag [19] geometries.

Due to the low gain, the multi-pass alexandrite amplifier design is pivotal for achieving energy enhancement with high extraction efficiency. A detailed numerical model of the diode-pumped amplifier is made as a function of crystal temperature, seed wavelength, pump pulse duration, and number of passes. Informed by experimental data of a four pass alexandrite 'diamond' amplifier, a $50 \mathrm{~mJ}$ diode-pumped alexandrite MOPA design is theoretically developed.

This work forms part of a proof-of-concept design for a diode-pumped alexandrite MOPA system for satellite-based lidar operation. However, an efficient alexandrite amplifier could be applicable in many other domains.

\section{Diode-side-pumped alexandrite slab amplifier design and modelling}

\subsection{Amplifier geometry}

The amplifier geometry used in this paper is a multi-pass diamond geometry. The design is based on a slab crystal which is side-pumped to provide a sheet of gain across the width $\left(w_{c}\right)$ and length $\left(L_{c}\right)$ of the slab. Different variations of multi-pass diamond amplifiers are shown in Figure 1. This is based on a combination of the grazing-incidence bounce geometry [18] and the folded zig-zag design [19]. The input beam incident at an end face undertakes a shallow total internal reflection (TIR) at the side face of the amplifier. This is reflected back into the amplifier with a mirror or coating on the back crystal face to form the 'diamond' shaped double-pass geometry. There are a large number of double-pass diamond configurations (denoted by $N$, see Figure 1a-c). After amplification from one double-pass diamond configuration $\left(N_{1}\right)$ the output can be redirected back into the crystal to form another double-pass diamond configuration $\left(N_{2}\right)$. This forms a four-pass amplifier (see Figure 1d). Further iterations could lead to six passes (see Figure 1e) or more, increasing gain and extraction.

The entrance angle $\left(\theta_{N}\right)$ and internal bounce angle $\left(\theta_{B}\right)$ are defined for a given diamond configuration $N$ (see Figure 1b, $\mathrm{N}=2$ for example), by:

$$
\begin{gathered}
\sin \theta_{N}=\frac{n N w_{c}}{\sqrt{N^{2} w_{c}^{2}+L_{c}^{2}}} \\
\tan \theta_{B}=\frac{N w_{c}}{L_{c}}
\end{gathered}
$$

given the crystal refractive index $n$, width $w_{c}$, and length $L_{c}$. The maximum number of possible diamonds is dictated by TIR at the entrance crystal face. This is given by:

$$
N_{\text {max }} \leq \frac{L_{c}}{w_{c} \sqrt{n^{2}-1}}
$$

for example, a $30 \mathrm{~mm}$ long crystal of width $2 \mathrm{~mm}$ and refractive index of 1.74 (for alexandrite) can sustain an $N_{\max }$ of 10 diamonds. The length of the crystal and the number of pump diodes can be scaled to produce the desired gain. In this design, we use double-pass side-pumping where the pump is incident on one slab side-face and reflected by a pump mirror at the opposite face. This improves the pump uniformity and efficiency by maximising the stored inversion density 


\section{Two Pass}

a)

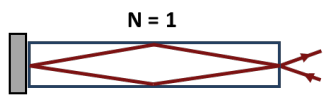

b)

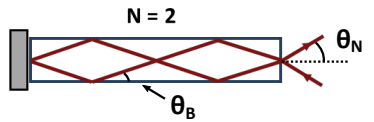

c)

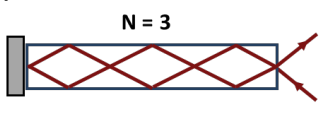

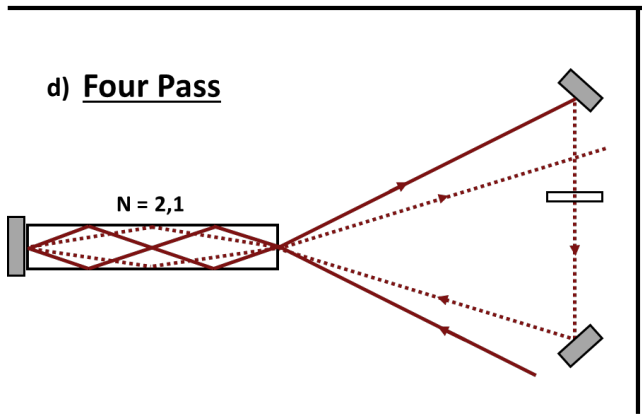

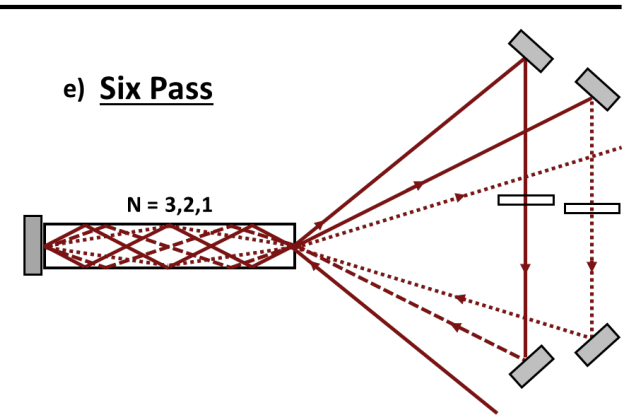

Fig. 1. Multi-pass amplifier concept. For a two-pass amplifier, the a) single $(\mathrm{N}=1), \mathrm{b})$ double $(\mathrm{N}=2)$, and $\mathrm{c})$ triple $(\mathrm{N}=3)$ diamond geometries are shown alongside potential four-pass (d), and six-pass (e) combinations. For the four-pass and six-pass configurations, additional HR (high-reflectance) mirrors and relay-image lenses are included.

from a single diode. This benefit, combined with a greater control on the number of passes, is ideal for an amplifier with low gain, increasing the extraction.

\subsection{Determining stored inversion within the amplifier}

The vibronically broadened electronic energy level structure of alexandrite is outlined in Figure 2 , alongside key optical transitions. Due to the vibronically broadened ground state $\left({ }^{4} \mathrm{~A}_{2}\right)$ and upper lasing state $\left({ }^{4} \mathrm{~T}_{2}\right)$, alexandrite acts as a four level laser with broad absorption and emission bands. Excited State Absorption (ESA) and Ground State Absorption (GSA) can also occur. In alexandrite, close proximity between the long-lived storage level $\left({ }^{2} \mathrm{E}\right)$ and short-lived vibronic level $\left({ }^{4} \mathrm{~T}_{2}\right)$ leads to enhanced Boltzmann filling of the vibronic level with temperature. This increases the emission cross section but reduces upperstate lifetime. When combined with the temperature and wavelength dependence of ESA and GSA, the accurate modelling of alexandrite requires complex considerations, as discussed further in this paper.

Due to the crystal structure of alexandrite, there are two possible dopant sites for the chromium ions, as defined by their symmetry. These are inversion sites and mirror sites [22]. The absorption and emission within alexandrite is dominated by mirror site ions. For synthetic alexandrite, $78 \pm$ $3 \%$ of the chromium dopant ions occupy these sites [22]. In this work, the mirror site ions are taken to be the active ion with total dopancy $N_{C r}$.

To determine the gain and extraction efficiency from an amplifier, the time-dependent stored inversion must be analysed. For a square pump pulse, the energy stored within the amplifier is given by:

$$
E_{\text {stored }}=h v A_{p} \int_{0}^{L_{p}} N_{2} d z=h v A_{p} N_{2} L_{p}
$$

where $h v$ denotes the laser photon energy, $N_{2}$ is the stored population inversion density, $A_{p}=h_{p} w_{c}$ is the gain cross sectional area where $h_{p}$ is the pump height, $w_{c}$ is the crystal width, and $L_{p}$ is the length of the pumped region. The population inversion density maintained at the end of the pump pulse, when the seed pulse is incident upon the amplifier, requires the analysis of 


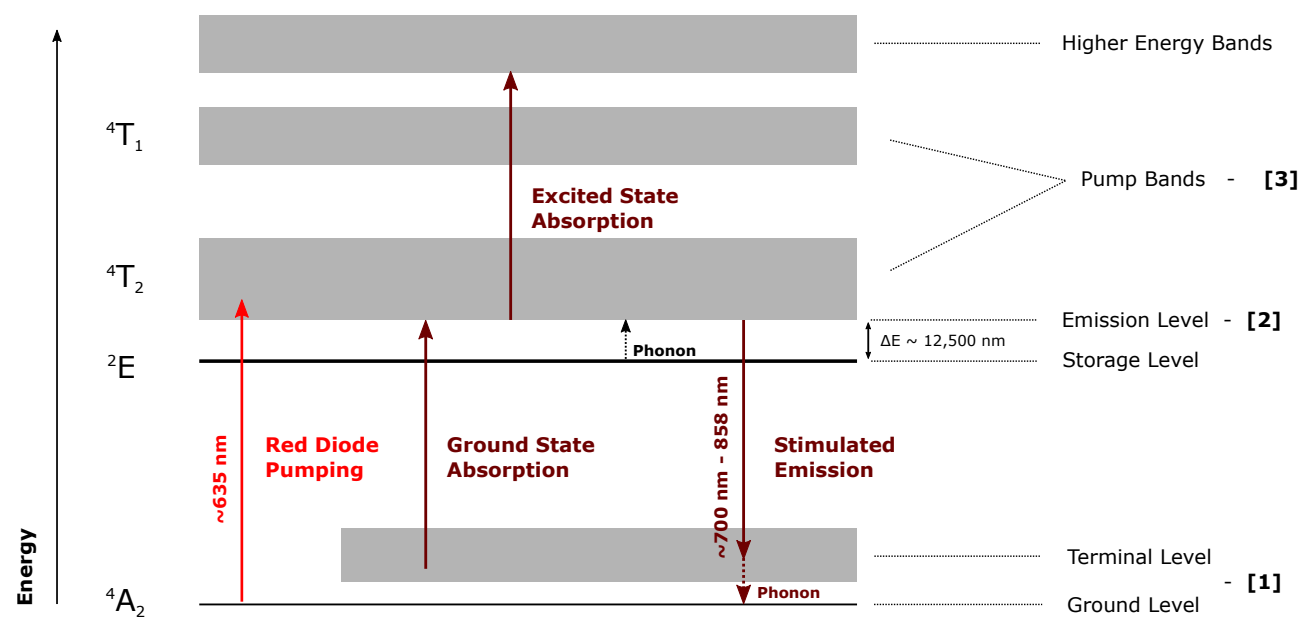

Fig. 2. The electronic energy level structure of alexandrite with the transitions responsible for ESA, GSA and stimulated emission. The vibronically broadening states are shown in grey. Based on [20] and [21].

multiple loss mechanisms. Assuming uniform inversion within the pump volume, $V_{p}=w_{c} h_{p} L_{p}$, the stored inversion density can be described by:

$$
N_{2}=\eta_{Q} \eta_{a b s} \eta_{21} \eta_{2} \frac{E_{p}}{E_{p h} V_{p}}
$$

where $\eta_{Q}$ is the pump quantum efficiency, taken to be 0.95 for alexandrite [23], $\eta_{a b s}$ is the absorption fraction of the incident double-passed pump photons, $\eta_{21}$ is an efficiency factor incorporating spontaneous emission loss, $\eta_{2}$ is a pump ESA efficiency factor [24], $E_{p}$ is the total incident pump energy, $V_{p}$ is the pump volume, and $E_{p h}$ is the energy of a single pump photon. The pump absorption efficiency, $\eta_{a b s}$ is calculated by considering the fraction of pump photons absorbed with the inclusion of the reflection losses of the pump faces.

The efficiency factor $\eta_{21}$ defines inversion depletion through spontaneous emission (fluorescence). This is defined as the ratio of the inversion remaining $\left(N_{2}\left(t=t_{p}\right)\right)$ to the total number of absorbed pump photons $\left(N_{p}\right)$, after a pump pulse of duration $t_{p}$, and is given by:

$$
\eta_{21}=\frac{N_{2}\left(t_{p}\right) V_{p}}{N_{p}}=\frac{\tau_{21}}{t_{p}}\left[1-e^{-\frac{t_{p}}{\tau_{21}}}\right]
$$

where $\tau_{21}$ is the upperstate lifetime.

The final storage efficiency loss mechanism, $\eta_{2}$, is the loss of pump photons through pump ESA from the upper lasing level, which is given by [24]:

$$
\eta_{2}=1-\gamma \bar{f}
$$

where $\bar{f}$ is the average fractional inversion when compared to the density of available mirror site ions $\left(N_{C r}\right)$, and $\gamma$ is the ratio of ESA cross section to GSA cross section, both at the pump wavelength. The fractional inversion is calculated using the average inversion:

$$
\overline{N_{2}}=\frac{1}{t_{p}} \int_{0}^{t_{p}} N_{2}(t) d t=\frac{N_{p} \tau_{21}}{t_{p}{ }^{2} V_{p}}\left[t_{p}+\tau_{21} e^{-\frac{t_{p}}{\tau_{21}}}-\tau_{21}\right]
$$

and the average fractional inversion is given by: 


$$
\bar{f}=\frac{\overline{N_{2}}}{N_{C r}}=\frac{\frac{N_{p} \tau_{21}}{t_{p}{ }_{2} V_{p}}\left[t_{p}+\tau_{21} e^{-\frac{t_{p}}{\tau_{21}}}-\tau_{21}\right]}{N_{C r}}
$$

For alexandrite, ions excited from the upperstate through pump ESA relax non-radiatively back to the same state [25]. Due to this, the effect of ESA is considered as a loss mechanism for pump absorption but does not deplete the inversion.

Modelling the stored inversion with crystal temperature determines the available gain and is discussed in Section 2.4, alongside the effects of seed wavelength. Efficient storage and extraction are key to producing an efficient MOPA system.

\subsection{Modelling amplification and gain extraction}

The alexandrite amplifier is modelled with pulsed pumping at a pulse rate low enough that the inversion returns to zero due to spontaneous emission before each consecutive pump pulse. After each pump pulse, the inversion reaches a level, $N_{2}$, when the seed enters the amplifier. For full modelling of the seed amplification, excited state absorption (ESA) and ground state absorption (GSA) at the laser wavelength are included alongside the passive losses. We consider both the small-signal amplification (low seed energy) when inversion depletion can be neglected, and the saturated regime (high seed energy) which is required for efficient energy extraction. The work in this section assumes a spatially uniform stored inversion across the pumped regions of the slab.

The small-signal gain factor, $G_{0}$, is given by

$$
G_{0}=\eta_{\text {loss }} \exp \left[\left(\sigma_{21^{*}} N_{2}-\sigma_{1} N_{1}\right) z_{p}-\sigma_{1} N_{C r} z_{n p}\right]
$$

where $\sigma_{21 *}=\sigma_{21}-\sigma_{2}$ is the effective emission cross section, $\sigma_{21}$ is the stimulated emission cross section, $\sigma_{2}$ is the ESA cross section at the laser wavelength, $\sigma_{1}$ denotes the GSA cross section at the laser wavelength, $N_{1}=N_{C r}-N_{2}$ is the ground state population density, and $z_{p}$ and $z_{n p}$ are the path lengths through the pumped and un-pumped regions of the amplifier, respectively. The passive losses of the amplifier such as scattering and reflection are included in $\eta_{\text {loss }}$. The first term in the exponential describes the pumped regions of the amplifier where the seed experiences small-signal gain alongside reduced GSA due to population inversion within the pump region. The final term in the exponential describes the GSA through the un-pumped regions of the amplifier where all the ions are in the ground state. To minimise losses the amplifier should be side-pumped along its entire length, reducing the effects of GSA (by reducing $z_{n p}$ to zero). Further gain is possible through optimisation of the seed wavelength and crystal temperature, maximising both extraction and energy storage, this is discussed in Section 2.4.

To maximise extraction in an amplifier, gain saturation should occur. To include the effects of saturation within the crystal a more involved method is necessary, the basis of which was developed by Frantz and Nodvik [26] and was later adapted for a zig-zag amplifier design by Eggleston et al. [27]. The latter includes the proportion of the pump region which is extracted by the zig-zag pass, ignoring regions not experienced by the beam. This gives:

$$
E_{\text {out }}=\eta E_{S} \cos \theta_{B} f(2-f) \cdot \ln \left\{1+\left[\exp \left[\frac{E_{\text {in }}}{E_{S} \cos \theta_{B} f(2-f)}\right]-1\right] \cdot \exp \left[\frac{E_{\text {stored }}}{E_{S} \cos \theta_{B}}\right]\right\}
$$

where $E_{s}=\left(\frac{h v}{\sigma_{21^{*}}} A\right)$ is the saturation energy of the amplifier, for the input mode area $A, f$ is the fill factor (width of the TIR on the pump face as a fraction of the distance between adjacent TIR bounces), $\theta_{B}$ is the bounce angle, $E_{\text {stored }}$ is the stored energy within the beam path and $E_{\text {in }}$ is the input pulse energy. The efficiency factor $\eta=\eta_{l o s s} \cdot \eta_{G S A}$ is a product of the passive losses $\eta_{\text {loss }}$ and GSA loss $\eta_{G S A}=\exp \left(-\sigma_{1} N_{1} z\right)$ for the path length, $z$, through the amplifier. The loss 
from ESA at the laser wavelength is accounted for with the use of an effective emission cross section $\sigma_{21^{*}}$.

This theory holds for the diamond geometry because this is an extension of the zig-zag geometry. One way to simplify the diamond bounce geometry for multi-pass amplification is to think of the diamond geometry as individual passes. To numerically model the extraction, the input pulse is divided into time increments of equal energy and propagated through the amplifier. The time increment size is chosen to allow its amplification within the small-signal regime. The gain on each pass and the extracted energy removed from the amplifier is calculated in a similar method to that utilised by Frantz and Nodvik [26].

\subsection{Effects of crystal temperature and seed wavelength on amplification}

For alexandrite, an increase in temperature leads to an effective increase in emission cross section. This is due to a higher fractional occupancy of the ${ }^{4} T_{2}$ state in the coupled upper-level ${ }^{4} T_{2}$ and ${ }^{2} E$ manifold. Figure 3 shows the wavelength-dependence of the cross sections for emission $\left(\sigma_{21}\right)$, ESA $\left(\sigma_{2}\right)$, and GSA $\left(\sigma_{1}\right)$ in alexandrite at three temperatures, $50{ }^{\circ} \mathrm{C}, 100{ }^{\circ} \mathrm{C}$, and $150{ }^{\circ} \mathrm{C}$. As shown, laser ESA has a minimum in the region of $750 \mathrm{~nm}-770 \mathrm{~nm}$ which is why four-level alexandrite lasers tend to operate in this wavelength region and not at shorter wavelengths where the emission cross section is higher. An increase in temperature leads to an increase in both the laser emission and ESA cross sections. This leads to a reduced increase in the effective emission cross section $\left(\sigma_{21^{*}}=\sigma_{21}-\sigma_{2}\right)$ with temperature, for longer wavelengths.

It is important to consider the trade-off between $\sigma_{21^{*}}$ and $\sigma_{1}$ with seed wavelength and temperature. GSA is reduced with longer seed wavelengths because the incoming photons cannot provide enough energy to excite electrons from the ground state. However, an increase in temperature will lead to an increase in occupancy of higher "ground state" sub-levels due to vibronic broadening and phonon interaction. This will lead to an increase in GSA loss because these broadened ground states contribute significantly to GSA.

Alongside variation in cross section, the fluorescence lifetime of Alexandrite reduces considerably with increased temperature [30]. The upperstate lifetime reduces from $262 \mu \mathrm{s}$ at $22{ }^{\circ} \mathrm{C}$ to around $150 \mu \mathrm{s}$ at $150{ }^{\circ} \mathrm{C}$ [31]. The effect driving this change is an increase in the occupation of the shorter $6.6 \mu$ s lifetime ${ }^{4} \mathrm{~T}_{2}$ state, reducing the occupancy of the longer $1.54 \mathrm{~ms}$ lifetime ${ }^{2} \mathrm{E}$ state [30]. This is covered in detail by Demirbas et al. [32]. The reduced upperstate lifetime reduces the stored energy within the amplifier, reducing the $\eta_{21}(T)$ efficiency factor. The temperature dependence of the fluorescence lifetime has been incorporated into the modelling throughout this paper.

To model the gain as accurately as possible, the temperature dependence of the absorption coefficient, $\alpha$, was measured for the $0.221 \%$ at. doped alexandrite slab used in experiments. The measured absorption coefficient ranged from $4.85 \mathrm{~cm}^{-1}$ to $5.70 \mathrm{~cm}^{-1}$ over the temperature range $40{ }^{\circ} \mathrm{C}$ to $150{ }^{\circ} \mathrm{C}$. In the simulation, this was accounted for in the efficiency factor, $\eta_{a b s}(T)$.

\section{Experimental results of master oscillator and four-pass 'diamond' amplifier}

\subsection{High-energy diode-pumped Q-switched alexandrite oscillator}

This section describes the results of a high-energy diode-pumped Q-switched alexandrite oscillator that would form the basis for the master-oscillator of a MOPA system. The cavity configuration is shown in the inset of Figure 4.

The oscillator consisted of a $0.221 \%$ at. doped alexandrite crystal pumped by two free-space red diode modules (used previously in [11], [12], [13]). The pump diodes were operated at 100 $\mathrm{Hz}$ with a pulse duration of $170 \mu \mathrm{s}$, producing a total available pump energy of $35 \mathrm{~mJ}$ at 635 $\mathrm{nm}$. After beam reshaping, a near-circular spot size of around $350 \mu \mathrm{m}$ (radius) was delivered in dual-end pumping as shown in the system schematic in the inset of Figure 4 . The $\mathrm{M}^{2}$ of the pump 


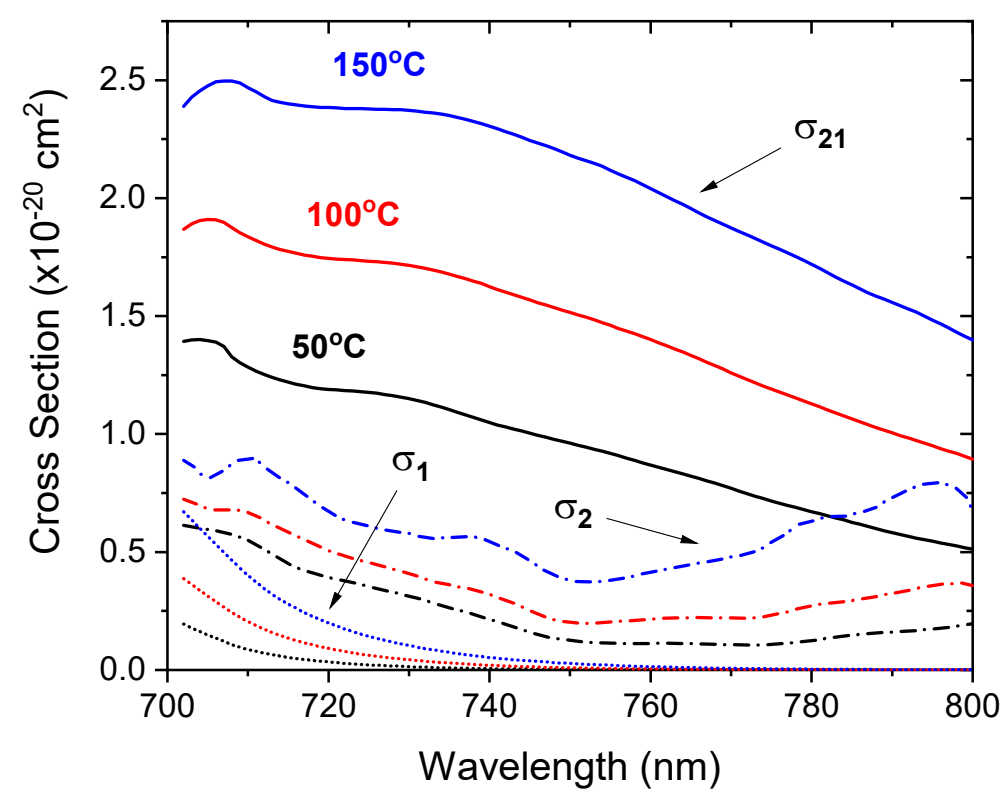

Fig. 3. The emission cross section $\left(\sigma_{21}\right)$, ESA cross section $\left(\sigma_{2}\right)$, and GSA cross section $\left(\sigma_{1}\right)$ with seed wavelength for $50{ }^{\circ} \mathrm{C}, 100{ }^{\circ} \mathrm{C}$ and $150{ }^{\circ} \mathrm{C}$ alexandrite crystal temperatures. The cross sections were all based on data and equations from Shand and Jenssen [28] and is further described in [29].

beam was approximately 175 and 60 in the vertical and horizontal, respectively. The crystal temperature was maintained at $55^{\circ} \mathrm{C}$.

The oscillator was Q-switched with a BBO Pockels cell. Lasing hold-off with the voltage across the Pockels cell was provided by a Birefringent Filter (BiFi) at Brewster's angle. This also acted to tune the wavelength of the cavity. A negative g-parameter cavity configuration was used consisting of an $\mathrm{f}=150 \mathrm{~mm}$ lens positioned close to its focal length away from the back mirror. The total cavity length was $270 \mathrm{~mm}$.

Figure 4 shows the laser pulse energy versus incident pump energy. The oscillator (with 93.1 $\%$ output coupler) produced a $3.32 \mathrm{~mJ}$ pulse of duration $88 \mathrm{~ns}$ at $100 \mathrm{~Hz}$, a central wavelength of $753 \mathrm{~nm}$, and a spatial beam quality with an $M_{x}^{2}$ of 2.00 and $M_{y}^{2}$ of 2.27 . The pulse energy was further increased to $3.80 \mathrm{~mJ}$ at $50 \mathrm{~Hz}$ with $220 \mu$ s pump pulse duration. This is thought to be due to a reduction in the crystal temperature from a reduced average pump power. The increased upperstate lifetime would improve the stored inversion between pulses. To the best of our knowledge, this is the highest pulse energy achieved from a diode-pumped Q-switched alexandrite oscillator.

\subsection{Experimental four-pass 'diamond' amplifier system}

The experimental amplifier system is outlined in Figure 5. The alexandrite slab is side-pumped by two diode modules (Stack A1 and Stack A2) that are retro-reflected to produce double-pass absorption. The seed enters the crystal to form an initial double-diamond $(\mathrm{N}=2)$ upon reflection near the back face (HR $755 \mathrm{~nm}$ ). The output is reshaped vertically and redirected to form a subsequent single diamond $(\mathrm{N}=1)$ on the second double-pass, forming a total of four passes 


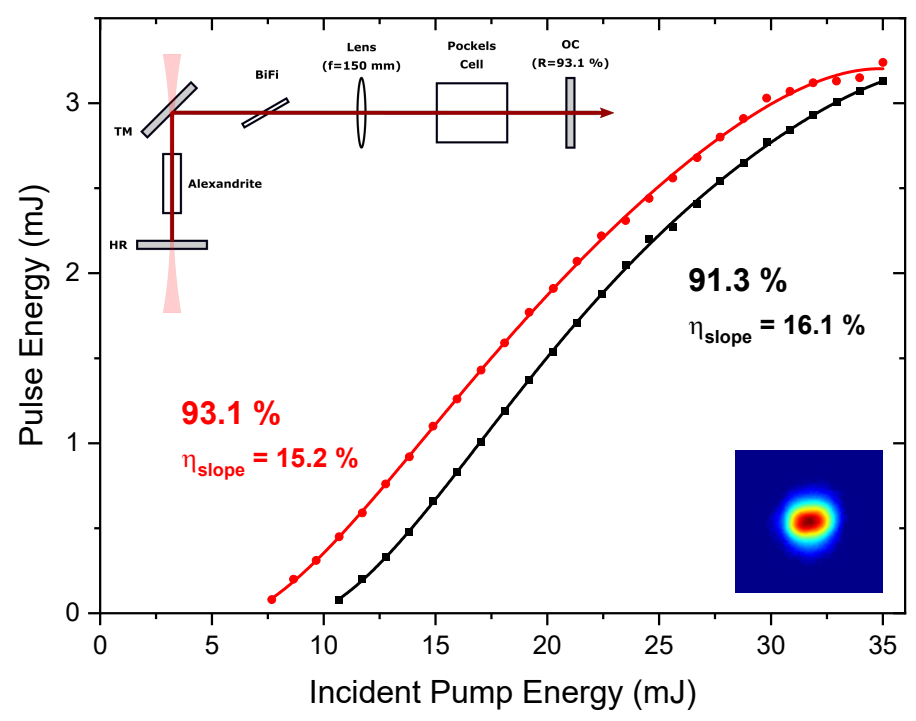

Fig. 4. Q-switched oscillator energy curves for output couplers (OC) with reflectivity 91.3 $\%$ and $93.1 \%$ at $755 \mathrm{~nm}$. The laser cavity configuration is shown in the top inset with the spatial output profile in the bottom right inset.

( $\mathrm{N}=2,1$ configuration). The beam shaping was optimised for maximum pump power.

The two diode pumps A1 and A2 were free-space red diode modules (previously used in [11], [12], [13]) operating at $635 \mathrm{~nm}$ and a pulse repetition rate of $100 \mathrm{~Hz}$. The combined incident pump energy for different pulse durations were $67 \mathrm{~mJ}$ (at $250 \mu \mathrm{s}$ ), $54 \mathrm{~mJ}$ (at $200 \mu \mathrm{s}$ ) and $40 \mathrm{~mJ}$ (at $150 \mu \mathrm{s}$ ). These modules side-pumped an alexandrite slab of dopant concentration 0.221 $\%$ at. and dimensions $30 \mathrm{~mm} \times 2 \mathrm{~mm} \times 2 \mathrm{~mm}$ (with an uncoated pump face). By focusing onto a plane mirror (HR at $636 \mathrm{~nm}$ ), the pump was retro-reflected to achieve a double-pass and increase absorption (around $78 \%$ at $40^{\circ} \mathrm{C}$ ), forming a near uniform inversion distribution across the width of the crystal. Across the width of the crystal the pump height varied from $180 \mu \mathrm{m}$ to 240 $\mu \mathrm{m}$, giving an average pump height of $h_{p}=210 \mu \mathrm{m}$. The total width of the two pump regions totalled $20 \mathrm{~mm}$, two-thirds of the crystal length. The crystal temperature was controlled with a TEC heater. The temperature of the crystal was recorded using a thermal camera, observing the upper face of the crystal. To determine the temperature of the pumped region of the slab, the experimentally measured system amplification with wavelength and temperature was compared with the theoretical behaviour (see Figure 8). The maximum recorded upper surface temperature was $133{ }^{\circ} \mathrm{C}$ which corresponded to an estimated pump region temperature of $150{ }^{\circ} \mathrm{C}$, as discussed in Section 3.3.

To test the performance of the amplifier, a variant on the diode-pumped Q-switched seed oscillator from Section 3.1 was constructed with very high beam quality. The output of this oscillator was tunable from $735 \mathrm{~nm}$ to $784 \mathrm{~nm}$ using a birefringent filter. The maximum output pulse energy was $0.55 \mathrm{~mJ}$ at $765 \mathrm{~nm}$ with a pulse duration of $114 \mathrm{~ns}$ and an $M^{2}$ beam quality of 1.06 (x) and 1.05 (y). High beam quality was maintained throughout the tuning range. For the amplified wavelengths of $745 \mathrm{~nm}$ and $770 \mathrm{~nm}$, the output pulse energy was $0.27 \mathrm{~mJ}$ and $0.42 \mathrm{~mJ}$, respectively. A seed mode size ( $\frac{1}{e^{2}}$ diameter) of $1300 \mu \mathrm{m}(\mathrm{x})$ and $164 \mu \mathrm{m}(\mathrm{y})$ was used at the amplifier input face to match the corresponding pump region. 


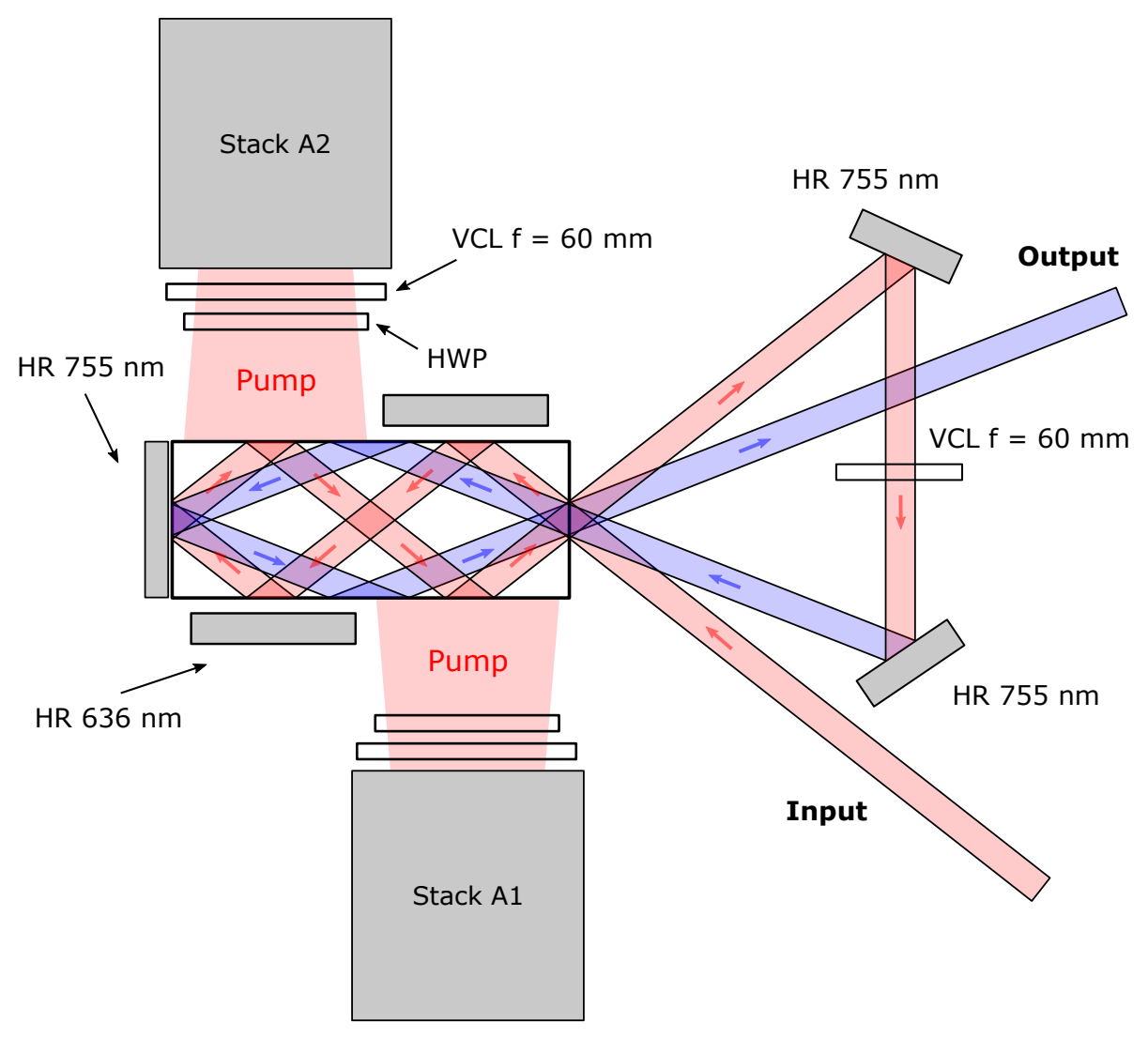

Fig. 5. Schematic of the experimental four-pass double-diamond and subsequent singlediamond ( $\mathrm{N}=2,1$ diamond geometry) amplifier system used in the study of the multi-pass amplifier concept.

\subsection{Results and Comparison}

The four pass diode-pumped alexandrite amplifier in Figure 5 was tested to assess the performance of the diamond geometry. The results of this performance assessment were later used to corroborate the simulated performance of the higher energy system described in Section 4.

The passive losses from the amplifier system were measured with a $780 \mathrm{~nm} \mathrm{CW}$ diode, giving a two-pass loss of $1.9 \%$ and a four-pass loss of $7.1 \%$. The loss is particularly high due to the non-optimal coatings of the crystal that were designed for $0^{\circ}$ incidence and the introduction of beam shaping optics for four passes. The seed experienced a high number of passes through these coatings at an angle away from the normal.

There are two definitions used in this paper to describe the amplifier performance: 'amplification' and 'system amplification'. The term 'amplification' is used to describe the ratio of the output of the amplifier with pumping relative to the output without pumping. This does not include the passive losses experienced by the seed. The term 'system amplification' is used in this work to describe the amplification of the output relative to the input and therefore includes passive losses and effects such as GSA and ESA at the laser wavelength. The experimental results of the seed oscillator amplification in the four-pass amplifier are shown in Figures 6-8 alongside results from simulations using Equation 11 and the methods outlined in Section 2.

The amplification and system amplification for the two-pass and four-pass amplifiers are shown in Figure 6, for both the experimental system (data points) and matched theoretical model (dashed 
lines) for seed wavelength $762 \mathrm{~nm}$, crystal temperature $150{ }^{\circ} \mathrm{C}$ and pump duration $250 \mu$ s. For the experimental amplifier, the two-pass $(\mathrm{N}=2)$ and four-pass $(\mathrm{N}=2,1)$ systems produced an amplification of 1.50 and 2.13, respectively. The corresponding two-pass and four-pass system amplification was 1.42 and 1.73 , respectively. The four pass amplification was also tested as a function of seed input energy, showing no significant variation. This suggests the amplifier was operating within the small-signal regime.

The beam quality of the amplifier output gave an $M^{2}$ of $1.31(\mathrm{x})$ and $3.20(\mathrm{y})$ for the double-pass and 2.18 (x) and $2.70(\mathrm{y})$ for the four-pass. The reduction in vertical (y) beam quality of the two-pass amplifier is thought to be due to an artefact in the beam as no diffractive clipping was visible. The output of the four-pass amplifier exhibited some diffractive clipping in the horizontal, suggesting the beam shaping was non-optimal (as confirmed by the beam quality measurements). This increased loss is shown in the system amplification of the four-pass amplifier (Figure 6).
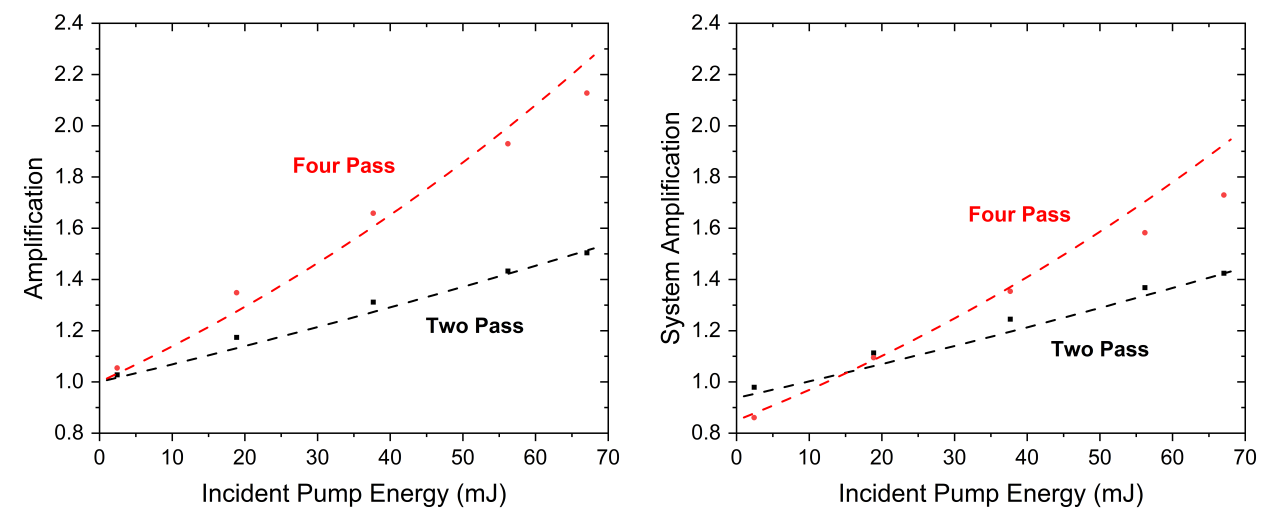

Fig. 6. Comparison of experimental results (data points) and theoretical simulation (dashed line) for four-pass (red) and two-pass (black) amplification (left) and system amplification (right) with a crystal temperature of $150{ }^{\circ} \mathrm{C}$, seed wavelength of $762 \mathrm{~nm}$, pulse energy of $0.55 \mathrm{~mJ}$, and pump pulse duration of $250 \mu \mathrm{s}$.

Figure 7 shows the impact of changing the pump pulse duration (250 $\mu$ s, $200 \mu$ s, and 150 $\mu \mathrm{s})$ on the two-pass system amplification both experimentally and in simulation, for a seed wavelength of $770 \mathrm{~nm}$. Decreasing the pump duration is seen to increase the efficiency of the amplifier (from $5.4 \mathrm{~J}^{-1}$ to $7.2 \mathrm{~J}^{-1}$ ) due to a reduction in spontaneous emission which increases $\eta_{21}$. However, reducing the pump pulse duration for a given diode will reduce the pulse energy available from that diode, unless over-driven. Experimental data is in reasonable qualitative agreement with the simulation results shown in Figure 7. The behaviour at low pump energy is thought to be due to seed mode-mismatch with a reduced thermal lens, given optimisation was at high pump energies.

Within alexandrite, the crystal temperature and seed wavelength are pivotal to efficient gain extraction. The seed wavelength exploits the spectral properties of the effective emission and GSA cross sections whilst the temperature acts to influence both these cross sections and the upperstate lifetime. The comparison between the experimental and theoretical behaviour of the amplifier with wavelength and temperature is shown in Figure 8 for three seed wavelengths. Whilst the gain generally increases with temperature, each seed wavelength has an optimum range of crystal temperature due to the increase in ground state absorption with temperature (see Figure 3). The increase in GSA with temperature was observable experimentally with the beam path within the amplifier being visible for crystal temperatures above $80^{\circ} \mathrm{C}$, due to high GSA and subsequent fluorescence. As shown in Figure 8, the shorter wavelength $745 \mathrm{~nm}$ seed 

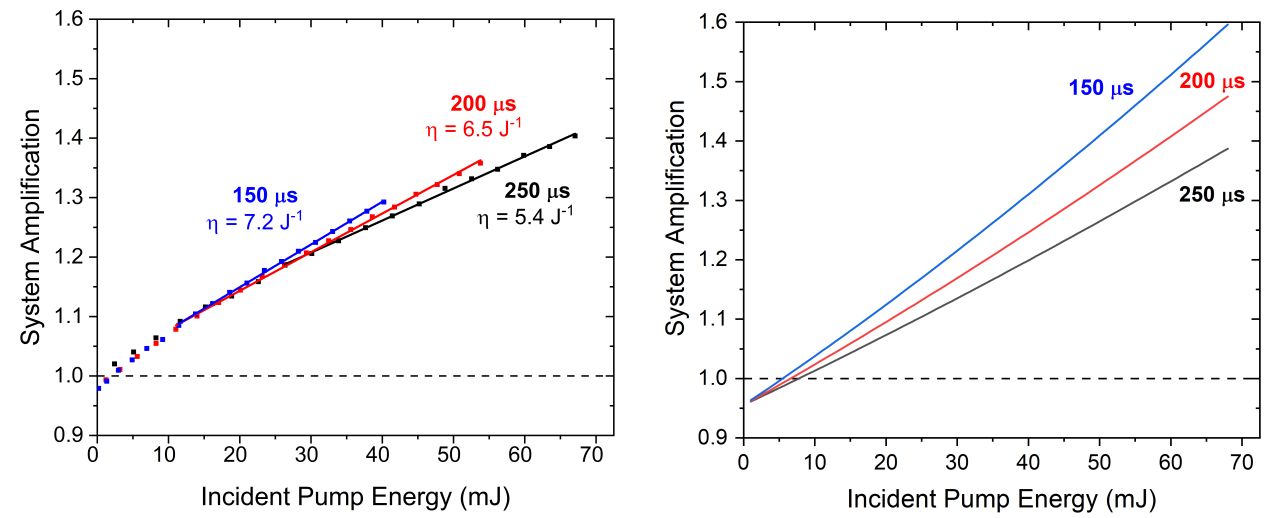

Fig. 7. Experimental data (left) and theoretical data (right) showing the change in two-pass system amplification with pump pulse duration for a $770 \mathrm{~nm}$ seed passing through a $150{ }^{\circ} \mathrm{C}$ crystal.

experiences 'roll-over' as the temperature is increased up to $150^{\circ} \mathrm{C}$ due to the onset of significant GSA. The general behaviour of the experimental system amplification with temperature and wavelength is in very good agreement with the simulated results, albeit with a temperature shift. This temperature shift was used to estimate the temperature of the pumped region of the crystal, as previously mentioned. The maximum measured surface temperature of $133{ }^{\circ} \mathrm{C}$ corresponded to an estimated pump region temperature of $150{ }^{\circ} \mathrm{C}$. The reduced experimental performance of the $745 \mathrm{~nm}$ seed is thought to be due to the spectral dependence of the optical coatings.
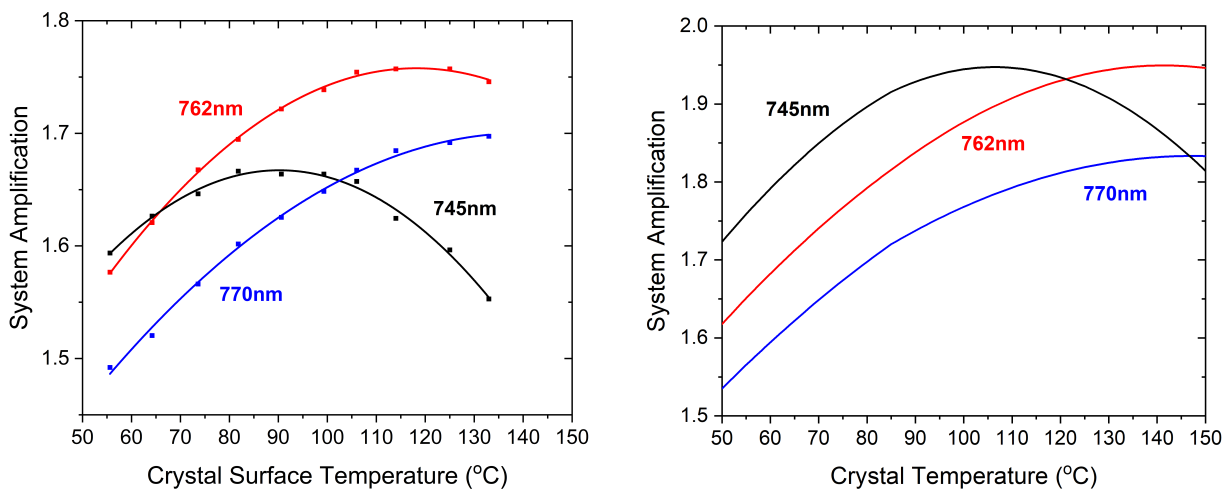

Fig. 8. Experimental data (left) and theoretical data (right) showing the four-pass system amplification with crystal temperature at maximum pump energy $(67 \mathrm{~mJ})$ for three seed wavelengths. The vertical axes differ in scale to aid visualisation of the graph behaviour.

Studying the fluorescence spectrum of the crystal showed the onset of a sharp feature at 751 $\mathrm{nm}$ for the highest pump levels due to parasitic lasing. Parasitic lasing involves oscillation within the slab based on total internal reflection from all four slab faces. This effect will act to reduce the gain available within the amplifier.

Due to the dimensions of the slab, the only diamond formations which allow parasitic lasing (TIR on all faces) are the $\mathrm{N}=11$ to $\mathrm{N}=21$ diamond configurations (see Equation 3). Our crystal was of a near perfect rectangular shape. This is not optimal, and future designs would include an angled input face to break the symmetry necessary for parasitic lasing to occur. This would not 
alter the internal diamond structure of the seed, only its external incident angle due to the change in the refracted angle at the entrance face.

\section{Proposed amplifier design for energy-scaled MOPA system}

Energy scaling will demonstrate the potential capability of high-energy $(50 \mathrm{~mJ})$ diode-pumped alexandrite systems. The work in this section is based on extending the theoretical simulations discussed previously. The target energies for the proposed MOPA design are outlined in Figure 9. The design consists of a two-stage amplifier (pre-amplifier and power amplifier) with an input of $5 \mathrm{~mJ}$ and an output target energy of $50 \mathrm{~mJ}$. This forms part of a proof-of-concept diode-pumped alexandrite MOPA design for atmospheric lidar.

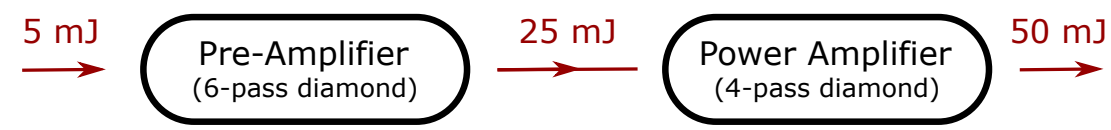

Fig. 9. Target pulse energies of the proposed diode-pumped alexandrite MOPA system.

The proposed pre-amplifier system is shown in Figure 10. The input face is angled to break the symmetry and prevent parasitic lasing. The amplifier is based on an input pulse energy of $5 \mathrm{~mJ}$ and a target output energy of $25 \mathrm{~mJ}$. The crystal is $40 \mathrm{~mm}$ in length and $2 \mathrm{~mm}$ in width with 0.221 $\%$ at. dopancy. The crystal is pumped by five commercially available 6-bar diode stack modules each with a peak power of $300 \mathrm{~W}$ at $636 \mathrm{~nm}$ providing a total peak power of $1500 \mathrm{~W}$. The pump produces a gain sheet of height $500 \mu \mathrm{m}$ across the entire crystal. The pump is retro-reflected at the other HR coated side-face to increase absorption and reduce the pump gradient across the crystal width. The total absorption is based on the temperature-dependent absorption coefficient measured experimentally.

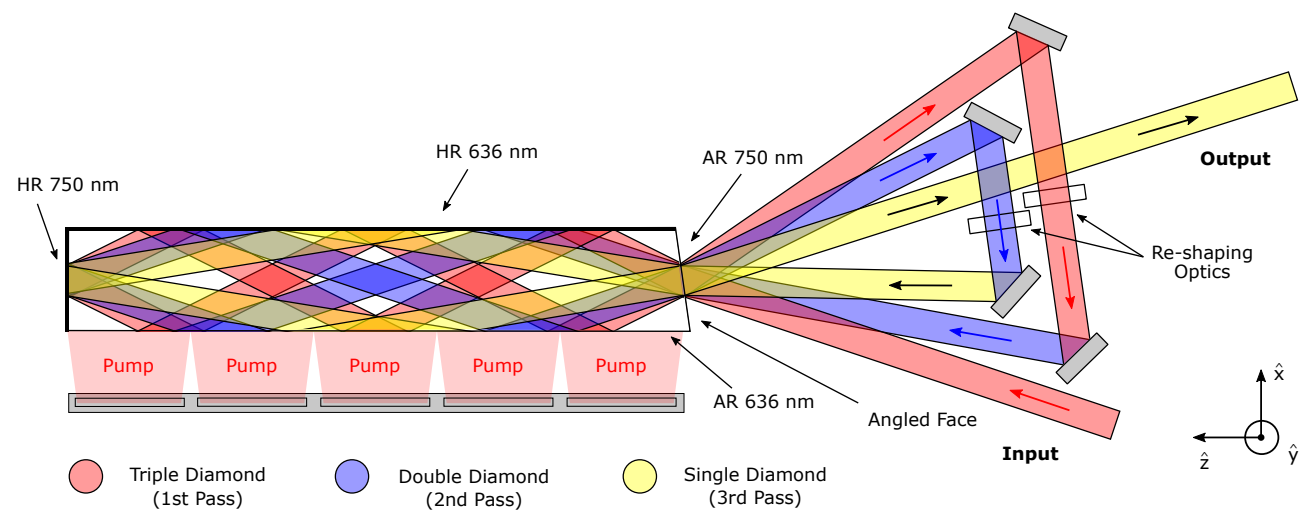

Fig. 10. Schematic of the pre-amplifier system consisting of a $40 \mathrm{~mm}$ alexandrite crystal side pumped by five diodes. The input seed takes an initial triple-diamond pass $(\mathrm{N}=3)$ followed by a double-diamond $(\mathrm{N}=2)$ and single-diamond $(\mathrm{N}=1)$ pass - experiencing six passes in total $(\mathrm{N}=3,2,1$ configuration).

The input seed mode size is taken to be $1500 \mu \mathrm{m}$ (x) by $400 \mu \mathrm{m}$ (y). The pump and input seed heights have been increased compared to the experimental setup in Section 3, in order to reduce the fluence on the crystal faces. The diamond geometry chosen is a six-pass system consisting of a triple, double and single diamond configuration $(\mathrm{N}=3,2,1)$ in that order (see Figure 10). Whilst a higher number of passes would increase the extraction, six passes were chosen to limit the 
fluence on the input face of the crystal. With optimised coatings, the reflection and scattering losses are estimated to be around $2 \%$ for the six-pass system.

The amplification calculation was performed using Equation 11 with the numerical modelling method described in Section 2.3. The interplay of the seed wavelength and temperaturedependence of the emission, ESA, GSA and upperstate lifetime were incorporated in the simulations, as previously described in Section 2.4 and illustrated by the cross section dependencies in Figure 3. To determine the optimum seed wavelength for the six-pass pre-amplifier, the output energy for different crystal temperatures and seed wavelengths was investigated for a pump pulse duration of $200 \mu \mathrm{s}$ (pump energy of $300 \mathrm{~mJ}$ with $1500 \mathrm{~W}$ peak pump power). Figure 11 shows the simulated results of the output energy of the six-pass diode-pumped amplifier versus seed wavelength for a range of temperatures from $25^{\circ} \mathrm{C}$ to $150^{\circ} \mathrm{C}$.

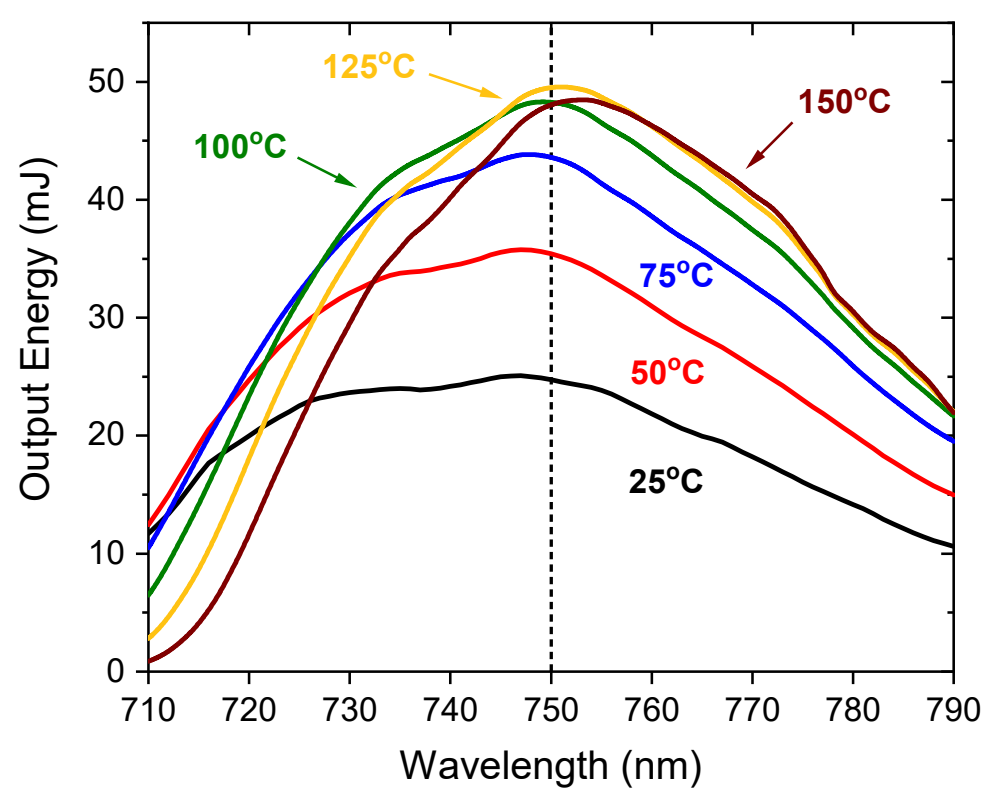

Fig. 11. The output energy of the six-pass amplifier with seed wavelength and crystal temperature. This is based on a $5 \mathrm{~mJ}$ input and a pump pulse duration of $200 \mu \mathrm{s}$. The near optimum $750 \mathrm{~nm}$ seed wavelength is highlighted. Based on a system with a $9.5 \%$ inversion fraction.

Figure 11 shows that high temperatures are in general favourable for enhancing longer wavelengths $(>750 \mathrm{~nm})$ due to enhanced emission cross section. However, at short wavelengths the optimum temperature decreases to avoid excessive ground state absorption. The maximum gain for the range of crystal temperatures used is found to be at a seed wavelength of around $750 \mathrm{~nm}$. This is due to the favourable combination of good emission cross section, minimum in excited state absorption and reduced ground state absorption at this wavelength.

The effect of pump pulse duration on the six-pass pre-amplifier with fixed peak pump power was investigated with a seed input of $750 \mathrm{~nm}$. The results of output energy versus pump pulse duration and crystal temperature are shown in Figure 12. As expected, an increased pump pulse duration results in increased amplification due to the greater pump energy. The optimal temperature decreases with increased pump pulse duration due to the reduced upper state lifetime at higher crystal temperatures which in turn reduces the storage efficiency. Whilst the simulation 
shows the pre-amplifier output energy up to $60 \mathrm{~mJ}$ with long pump pulse durations, the output energy is limited by operation within a safe margin below the laser-induced damage threshold of the crystal coatings. This requires a lower output pulse energy of approximately $25 \mathrm{~mJ}$. Additionally, it is important to consider extraction efficiency, which favours operation at a shorter pump pulse duration and higher temperature.

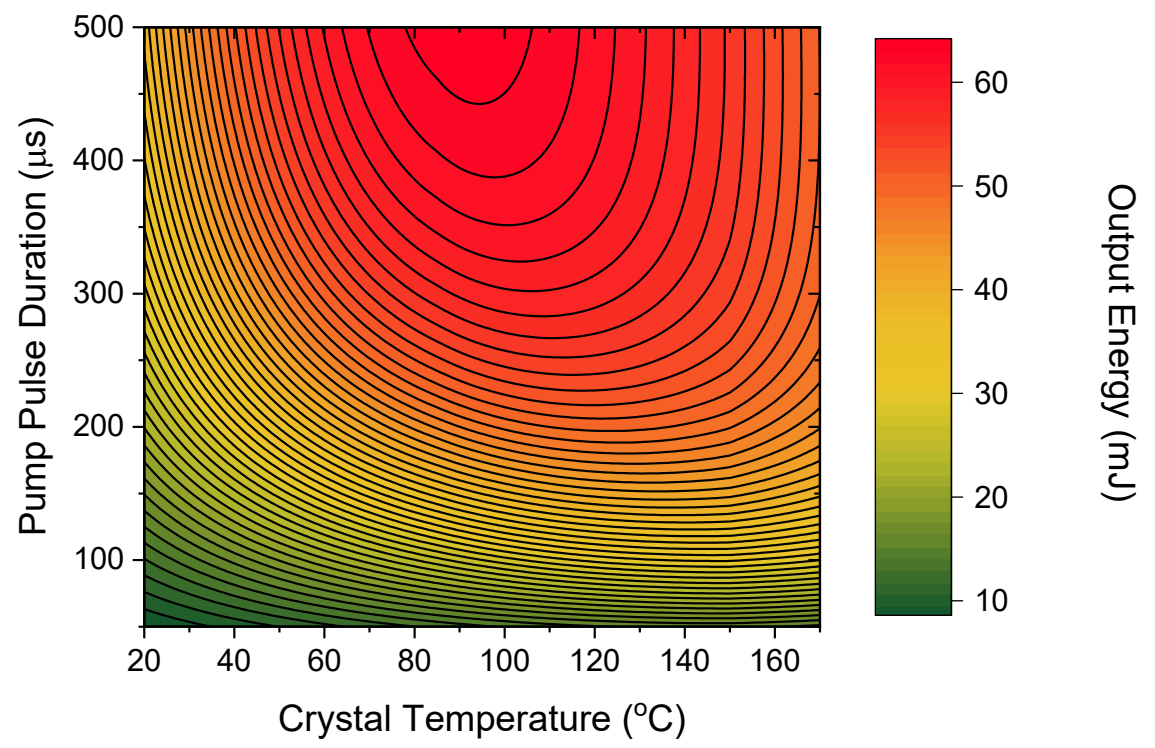

Fig. 12. The variation in six-pass pre-amplifier output energy with pump pulse duration and crystal temperature for a $5 \mathrm{~mJ}, 750 \mathrm{~nm}$ input seed.

The modelling simulation results of Figure 12 suggest that the optimum extraction efficiency which achieves an output of $25 \mathrm{~mJ}$ occurs at a pump pulse duration of $80 \mu \mathrm{s}$ and crystal temperature of $140^{\circ} \mathrm{C}$. For operation at this pulse duration, the pump diodes with peak power $1500 \mathrm{~W}$ produce $120 \mathrm{~mJ}$ of incident pump energy at $636 \mathrm{~nm}$. The pump diodes produce a gain sheet with cross sectional dimensions of $40 \mathrm{~mm} \times 2 \mathrm{~mm}$ and pump height of $500 \mu \mathrm{m}$. The input seed has a mode size of $1.5 \mathrm{~mm}$ (x) x $400 \mu \mathrm{m}$ (y), with a wavelength of $750 \mathrm{~nm}$. The $5 \mathrm{~mJ}$ input pulse of nominal duration $100 \mathrm{~ns}$, is amplified to $25 \mathrm{~mJ}$ within six passes of the diamond geometry $(\mathrm{N}=3,2,1)$.

The proposed power amplifier is designed to have an input of $25 \mathrm{~mJ}$ coming from the preamplifier and an output target energy of $50 \mathrm{~mJ}$. To operate within acceptable damage fluence limits at this higher energy, the power amplifier is designed for a larger seed input mode size of 3 $\mathrm{mm}(\mathrm{x}) \mathrm{x} 400 \mu \mathrm{m}$ (y) and restricted to a four-pass geometry ( $\mathrm{N}=2,1$ diamond configuration). To accommodate the larger mode size the power amplifier alexandrite slab has a larger width of 4 $\mathrm{mm}$ and is pumped on each side by three 6-bar diode modules. The slab length is shortened to 24 $\mathrm{mm}$ to reduce GSA. The pump provides a gain sheet of height $500 \mu \mathrm{m}$ across the $4 \mathrm{~mm}$ width and $24 \mathrm{~mm}$ length of the slab.

The same temperature and pump pulse duration optimisation was completed for the power amplifier with the $750 \mathrm{~nm}$ input seed. The results are shown in Figure 13. The maximum output energy of the power amplifier is at a higher temperature than the pre-amplifier (see Figure 12). This is due to the reduced ground state absorption from a shorter seed path length through the crystal. To efficiently achieve the $50 \mathrm{~mJ}$ target, analysis of Figure 13 suggests a crystal 
temperature of $125^{\circ} \mathrm{C}$ alongside a pump pulse duration of $90 \mu$ s corresponding to $162 \mathrm{~mJ}$ of incident pump energy at $636 \mathrm{~nm}$.

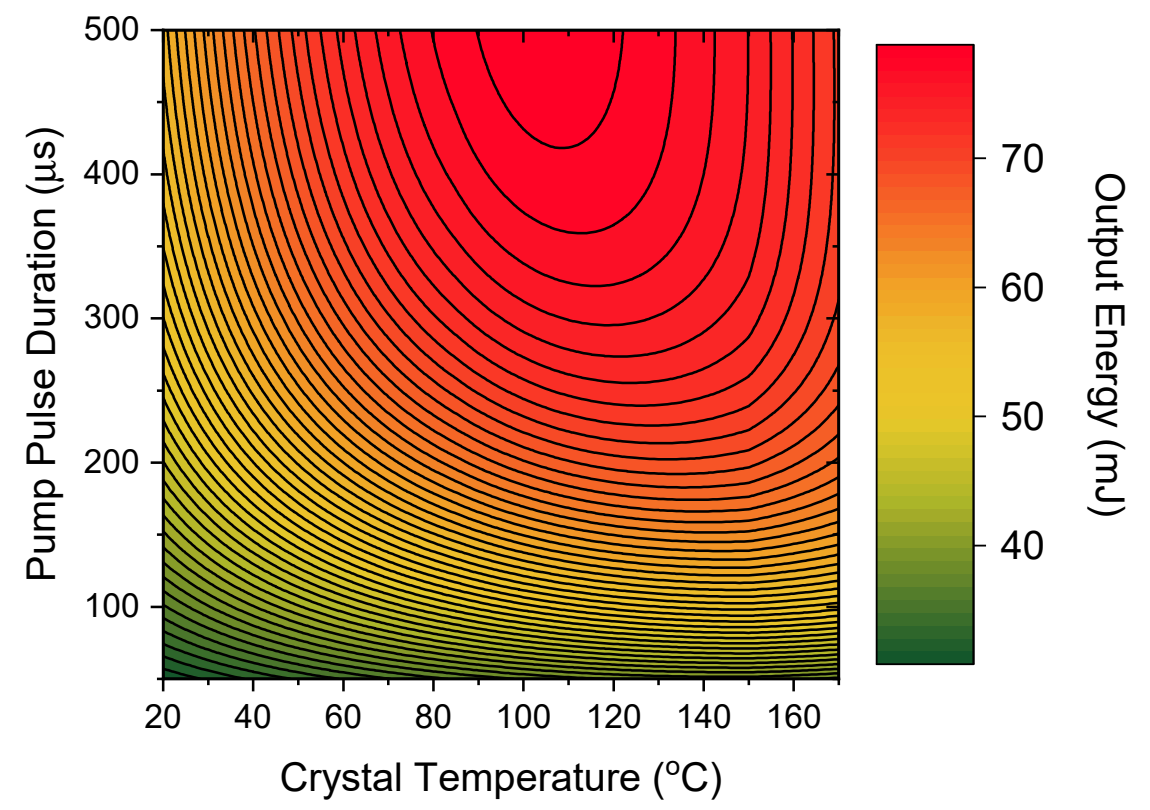

Fig. 13. The output energy of the four-pass power amplifier with pump pulse duration and crystal temperature for a $25 \mathrm{~mJ}$ input energy at $750 \mathrm{~nm}$.

In summary, for the pre-amplifier and power amplifier, the optical efficiency (extracted energy as a fraction of incident pump energy) was $16.7 \%$ and $15.4 \%$, respectively. The corresponding inversion fractions $\left(N / N_{C r}\right)$ maintained at the time of input seed extraction were $9.5 \%$ and $10.5 \%$. Comparing extracted energy to available stored energy, the extraction efficiency of the pre-amplifier and power amplifiers were $32.8 \%$ and $24.9 \%$. The greater extraction efficiency of the pre-amplifier is due to the greater number of passes (six compared to four). For the combined pre-amplifier and power-amplifier system, the overall optical efficiency is $16.0 \%$ and the extraction efficiency is $27.9 \%$.

This current design is for $750 \mathrm{~nm}$. A further design at $770 \mathrm{~nm}$ would be desirable to target the absorption line of potassium within the upper mesosphere and lower thermosphere enabling resonance scattering lidar. Following a similar optimisation at $770 \mathrm{~nm}$ based on the same amplifier design, the pre-amplifier pump diodes would operate with a pulse duration of $90 \mu \mathrm{s}$, producing a total pump energy of $135 \mathrm{~mJ}$ and the power amplifier diodes would operate at 100 $\mu \mathrm{s}$, producing a total pump energy of $180 \mathrm{~mJ}$. The crystal temperatures would be increased to $160{ }^{\circ} \mathrm{C}$ given the reduced ground state absorption at $770 \mathrm{~nm}$. This design would enable $50 \mathrm{~mJ}$ to be achieved from an initial $5 \mathrm{~mJ}$ seed oscillator with an overall optical efficiency of $14.3 \%$.

\section{Conclusion}

Alexandrite laser systems are wavelength tunable with high pulse energy capability but due to inefficient lamp-pumping have been limited in commercial application, predominantly to the field of cosmetic surgery. Recent work with red diode-pumping of alexandrite with low quantum defect has demonstrated its potential as a versatile high efficiency tunable laser material providing 
an opportunity for new scientific and commercial applications. Q-switched diode-pumped alexandrite laser oscillators have been demonstrated, but operation has not been far above the 1 $\mathrm{mJ}$ level, and this is insufficient for many applications - including satellite-based atmospheric remote sensing where higher energies towards the $100 \mathrm{~mJ}$-class are desirable.

In this paper, we developed a high-energy scaling strategy for diode-pumped alexandrite using a master-oscillator power-amplifier (MOPA) approach. A novel diamond geometry slab amplifier scheme was introduced allowing scalable side-pumping by low brightness high-power diode pump modules combined with the ability to scale the number of passes. The multi-pass capacity of the diamond geometry is ideal for materials such as alexandrite with low emission cross section. In this work, experimental demonstrations at moderate energy were performed alongside a full numerical design and optimisation of the MOPA for high-energy scaling.

In experimental work, a diode-pumped alexandrite oscillator was constructed producing a record Q-switched pulse energy of $3.80 \mathrm{~mJ}$. The first diode-pumped alexandrite amplifier was also demonstrated. Based on a four-pass diamond geometry, a series of diode-pumped alexandrite amplifier experiments were made as a function of diode pump energy and amplifier crystal temperature at multiple seed wavelengths. An amplification of 2.13 (system amplification of 1.73) was achieved at $762 \mathrm{~nm}$. Good qualitative agreement of amplifier results was found with theory, although seed mode-mismatch and the onset of parasitic lasing in the four-pass amplifier was identified. Elimination of these effects would further improve the results.

A detailed numerical analysis was performed on an energy-scaled alexandrite MOPA design with consecutive multi-pass diamond geometry amplifiers. Optimisation was achieved as a function of crystal temperature and pump pulse duration for different seed wavelengths. A $50 \mathrm{~mJ}$ diode-pumped alexandrite MOPA design at $750 \mathrm{~nm}$ had an overall expected optical efficiency of $16.0 \%$ (amplifier extraction efficiency of $27.9 \%$ ); at $770 \mathrm{~nm}$ (suitable for potassium resonance scattering lidar) the overall expected optical efficiency was $14.3 \%$. Further efficiency improvements are possible, which, together with advancements in diode technology, will support alexandrite as a viable space-compliant laser for future satellite-based remote sensing missions requiring tunability in the near infrared and the ultraviolet (with harmonic conversion).

\section{Acknowledgements}

The authors thank M. Kehoe and S. Johnson from the Imperial College Optomechanical Workshop for building bespoke parts for this work. A. Coney thanks G. M. Thomas and A. Minassian for their guidance in this work.

\section{Funding}

The authors gratefully acknowledge the funding of the European Space Agency in this project (Contract No. 4000115840/NL/RA/ZK) and Alexander T. Coney gratefully acknowledges the funding of an EPSRC development for Remote Sensing Award (1732149).

Engineering and Physical Sciences Research Council (1732149)

\section{Disclosures}

The authors declare no conflicts of interest.

\section{References}

1. A. Cosentino, A. D'Ottavi, A. Sapia, and E. Suetta, "Spaceborne lasers development for Aladin and Atlid instruments," in IEEE International Geoscience and Remote Sensing Symposium, (2012), pp. 5673-5676.

2. J. Walling, D. Heller, H. Samelson, D. Harter, J. Pete, and R. Morris, "Tunable alexandrite lasers: development and performance,” IEEE J. Quantum Electron. 21, 1568-1581 (1985). 
3. J. Kuper, T. Chin, and H. Aschoff, "Extended tuning range of alexandrite at elevated temperatures," in Advanced Solid State Lasers, (Optical Society of America, 1990), p. CL3.

4. C. L. Korb, G. K. Schwemmer, M. Dombrowski, and C. Y. Weng, "Airborne and ground based lidar measurements of the atmospheric pressure profile," Appl. optics 28, 3015-3020 (1989).

5. E. V. Browell, T. D. Wilkerson, and T. J. McIlrath, "Water vapor differential absorption lidar development and evaluation," Appl. optics 18, 3474-3483 (1979).

6. P. Ponsardin, N. S. Higdon, B. E. Grossmann, and E. V. Browell, "Spectral control of an alexandrite laser for an airborne water-vapor differential absorption lidar system," Appl. optics 33, 6439-6450 (1994).

7. U. von Zahn and J. Höffner, "Mesopause temperature profiling by potassium lidar," Geophys. research letters 23, 141-144 (1996).

8. J. Lautenbach, J. Hoeffner, P. Menzel, and P. Keller, "The new scanning iron lidar, current state and future developments," Sandefjord 5, 327-329 (2005).

9. A. Munk, M. Strotkamp, M. Walochnik, B. Jungbluth, M. Traub, H.-D. Hoffmann, R. Poprawe, J. Höffner, and F.-J. Lübken, "Diode-pumped Q-switched alexandrite laser in single longitudinal mode operation with watt-level output power," Opt. letters 43, 5492-5495 (2018).

10. M. Alpers, R. Eixmann, C. Fricke-Begemann, M. Gerding, and J. Höffner, "Temperature lidar measurements from 1 to $105 \mathrm{~km}$ altitude using resonance, Rayleigh, and rotational Raman scattering," Atmospheric Chem. Phys. 4, 793-800 (2004).

11. M. Damzen, G. Thomas, and A. Minassian, "Diode-side-pumped alexandrite slab lasers," Opt. express 25, 1162211636 (2017).

12. G. Thomas, A. Minassian, X. Sheng, and M. Damzen, "Diode-pumped alexandrite lasers in Q-switched and cavity-dumped Q-switched operation,” Opt. express 24, 27212-27224 (2016).

13. A. Teppitaksak, A. Minassian, G. M. Thomas, and M. J. Damzen, "High efficiency $>26 \mathrm{~W}$ diode end-pumped alexandrite laser," Opt. express 22, 16386-16392 (2014).

14. M. Strotkamp, A. Munk, B. Jungbluth, H.-D. Hoffmann, and J. Höffner, "Diode-pumped alexandrite laser for next generation satellite-based earth observation lidar," CEAS Space J. 11, 413-422 (2019).

15. P. Pichon, A. Barbet, J.-P. Blanchot, F. Druon, F. Balembois, and P. Georges, "LED-pumped alexandrite laser oscillator and amplifier," Opt. letters 42, 4191-4194 (2017).

16. G. Tawy, J. Wang, and M. J. Damzen, "Pump-induced lensing effects in diode pumped alexandrite lasers," Opt. Express 27, 35865-35883 (2019).

17. A. Munk, B. Jungbluth, M. Strotkamp, H.-D. Hoffmann, R. Poprawe, J. Höffner, and F.-J. Lübken, "Diode-pumped alexandrite ring laser in single-longitudinal mode operation for atmospheric lidar measurements," Opt. express 26, 14928-14935 (2018).

18. J. Bernard and A. Alcock, "High-efficiency diode-pumped Nd:YVO 4 slab laser," Opt. Lett. 18, 968-970 (1993).

19. J. Richards and A. McInnes, "Versatile, efficient, diode-pumped miniature slab laser," Opt. letters 20, 371-373 (1995).

20. R. C. Powell, L. Xi, X. Gang, G. J. Quarles, and J. C. Walling, "Spectroscopic properties of alexandrite crystals," Phys. Rev. B 32, 2788 (1985).

21. M. Fibrich, J. Šulc, D. Vyhlídal, H. Jelínková, and M. Čech, "Alexandrite spectroscopic and laser characteristic investigation within a 78-400 K temperature range," Laser Phys. 27 (2017).

22. C. Forbes, "Analysis of the spin-hamiltonian parameters for $\mathrm{Cr}^{3+}$ in mirror and inversion symmetry sites of alexandrite $\left(\mathrm{Al}_{2-x} \mathrm{Cr}_{x} \mathrm{BeO}_{4}\right)$. determination of the relative site occupancy by EPR," The J. chemical physics 79, 2590-2599 (1983).

23. M. L. Shand, "Quantum efficiency of alexandrite," J. applied physics 54, 2602-2604 (1983).

24. W. Kerridge-Johns and M. Damzen, "Analysis of pump excited state absorption and its impact on laser efficiency," Laser Phys. Lett. 12, 125002 (2015).

25. M. Shand, J. Walling, and R. Morris, "Excited-state absorption in the pump region of alexandrite," J. Appl. Phys. 52, 953-955 (1981).

26. L. M. Frantz and J. S. Nodvik, "Theory of pulse propagation in a laser amplifier," J. Appl. Phys. 34, 2346-2349 (1963).

27. J. Eggleston, L. Frantz, and H. Injeyan, "Deviation of the Frantz-Nodvik equation for zig-zag optical path, slab geometry laser amplifiers," IEEE J. Quantum Electron. 25, 1855-1862 (1989).

28. M. Shand and H. Jenssen, "Temperature dependence of the excited-state absorption of alexandrite," IEEE J. Quantum Electron. 19, 480-484 (1983).

29. W. R. Kerridge-Johns and M. J. Damzen, "Analytical model of tunable alexandrite lasing under diode end-pumping with experimental comparison," JOSA B 33, 2525-2534 (2016).

30. J. Walling, O. Peterson, H. Jenssen, R. Morris, and E. O’Dell, “Tunable alexandrite lasers,” IEEE J. Quantum Electron. 16, 1302-1315 (1980).

31. I. Yorulmaz, E. Beyatli, A. Kurt, A. Sennaroglu, and U. Demirbas, "Efficient and low-threshold alexandrite laser pumped by a single-mode diode," Opt. Mater. Express 4, 776-789 (2014).

32. U. Demirbas, A. Sennaroglu, and F. X. Kärtner, "Temperature dependence of alexandrite effective emission cross section and small signal gain over the $25-450^{\circ} \mathrm{C}$ range,” Opt. Mater. Express 9, 3352-3370 (2019). 